\title{
Policy and Law Assessment of COVID-19 Based on Smooth Transition Autoregressive Model
}

\author{
Jieqi Lei, ${ }^{1}$ Xuyuan Wang, ${ }^{2}$ Yiming Zhang, ${ }^{3}$ Lian Zhu $\mathbb{D}^{4},{ }^{4}$ and Lin Zhang $\mathbb{D}^{2}$ \\ ${ }^{1}$ School of Humanities, Beijing University of Posts and Telecommunications, Beijing 100876, China \\ ${ }^{2}$ School of Science, Beijing University of Posts and Telecommunications, Beijing 100876, China \\ ${ }^{3}$ International School, Beijing University of Posts and Telecommunications, Beijing 1000876, China \\ ${ }^{4}$ School of Journalism and Communication, Shanghai International Studies University, Shanghai 200083, China \\ Correspondence should be addressed to Lian Zhu; zhulian@shisu.edu.cn and Lin Zhang; zhanglin2011@bupt.edu.cn
}

Received 16 October 2020; Revised 4 December 2020; Accepted 22 December 2020; Published 20 January 2021

Academic Editor: Xiaoke Xu

Copyright $\odot 2021$ Jieqi Lei et al. This is an open access article distributed under the Creative Commons Attribution License, which permits unrestricted use, distribution, and reproduction in any medium, provided the original work is properly cited.

\begin{abstract}
As of the end of October 2020, the cumulative number of confirmed cases of COVID-19 has exceeded 45 million and the cumulative number of deaths has exceeded 1.1 million all over the world. Faced with the fatal pandemic, countries around the world have taken various prevention and control measures. One of the important issues in epidemic prevention and control is the assessment of the prevention and control effectiveness. Changes in the time series of daily new confirmed cases can reflect the impact of policies in certain regions. In this paper, a smooth transition autoregressive (STAR) model is applied to investigate the intrinsic changes during the epidemic in certain countries and regions. In order to quantitatively evaluate the influence of the epidemic control measures, the sequence is fitted to the STAR model; then, comparisons between the dates of transition points and those of releasing certain policies are applied. Our model well fits the data. Moreover, the nonlinear smooth function within the STAR model reveals that the implementation of prevention and control policies is effective in some regions with different speeds. However, the ineffectiveness is also revealed and the threat of a second wave had already emerged.
\end{abstract}

\section{Introduction}

In 2020, the COVID-19 epidemic is changing the way how people live, work, study, and socialize [1,2]. In the absence of effective vaccines, only nonpharmaceutical public health interventions can be used to break the chain of transmission of the virus $[3,4]$. Huge social and economic cost measures are conducted, such as the "lockdown" of severe regions, the establishment of adequate isolation places, the closure of nonessential public venues, and the closure of schools and workplaces [5-10].

China has adopted a series of strict control measures, including the lockdown of Wuhan starting from January 23, 2020, the establishment of Vulcan Mountain Hospital, Thor Mountain Hospital, and mobile cabin hospital, calling for the public to stay at home, not dining together, not visiting friends and relatives, working from home for adults, studying online at home for kids and college students, and tracing and isolating close contacts. The virus spread alarmingly fast in late January in China. Based on the public reports on the number of confirmed cases, the prevalence of COVID-19 outside Hubei Province came to a controllable size in late February [11-14]. The epidemic has been under effective control by March 2020. The Wuhan lockdown eventually ended on April 8, 76 days since its commencement. Moreover, China has timely contained several scattered confirmed cases in Jilin, Beijing, Xinjiang, Liaoning, and other places afterwards.

During the pandemic, all countries have implemented various prevention and control policies. Faced with a novel coronavirus that is highly contagious without reliable vaccine, the effectiveness of policies in different countries and regions is an important and urgent topic. Gregory et al. [15] provided evidence that state-wide mandates are effective in promoting social distancing in America through Google big data. Saez et al. [16] used a time-series design and a 
generalized linear hybrid model to explain and estimate the changes in the epidemic curve following quarantine measures in Spain. Nicholas et al. [17] used a random age structure propagation model to explore the effects of a range of interventions in UK and found that all four basic interventions were likely to reduce the basic reproductive number. It has been shown that in most countries the number of daily confirmed cases and the number of daily deaths show signs of decline from 1 to 4 weeks after the highest levels of social distancing measures were enacted and that the effect of social distancing measures on COVID-19 transmission varies among countries [18].

The effectiveness of prevention and control measures will be directly reflected in the trend of the epidemic. In order to quantitatively identify the inflection points hidden in the time series of daily new confirmed cases and further evaluate the effectiveness, a smooth transition autoregressive (STAR) model is utilized to analyze the epidemic data in different countries and regions.

Transitions are usually characterized by nonlinear models [19-21]. There are three main types of nonlinear models: the ARCH model proposed by Engle [22], the Markov mechanism transition model proposed by Hamilton with discrete variations of dependent variables determined by Markov chain [23], and the smooth transition autoregressive model proposed by Granger and Terasvirta [24], namely, the STAR model, while Tong's autoregressive model of the gate can be regarded as a special case of STAR [25]. ARCH model is mainly to describe the volatility of nonlinear. The Markovian transition model needs much information to infer the state of the variables, and the result can only infer the probability of the transformation zone, without specifically describing the nonlinear form of the transformation. The STAR model can realize the continuous smooth nonlinear transition and gives the nonlinear form of the mechanism transformation. Compared with other models, our method can not only well fit the epidemic curve but also quantify the inflection point, as well as the speed of transition, of the epidemic time series without external or prior information.

Combining with the release time of policies, we can provide the evaluation of the effectiveness of epidemic prevention and control measures. In fact, the inflection points are related to the quantitative parameters reflecting the switch of expansion and contraction within the sequences. It is revealed by the nonlinear function in the STAR model that the effectiveness of certain policies usually showed up within 2 weeks to 2 months. Ineffective policies have little impact on the trend of time series within 2 months or more.

An outline of this paper is as follows. The data and methodology are given in Section 2. Results are illustrated in Section 3. Discussions are given in Section 4.

\section{Data and Methodology}

The daily confirmed cases of COVID-19 in different regions, which are Guangdong Province in China, Brazil, France, Germany, India, Italy, Mexico, Spain, and the United States of America, are collected from the World Health Organization database. Before fitting to our model, the original time series are smoothed in order to reduce the fluctuation and keep the trend. In addition, measures and policies to prevent the transmission of COVID-19 in different regions are collected from national or regional official websites and news websites. The information and time series of daily confirmed cases are combined together to analyze the relationship between the social measures and the intrinsic changes of daily confirmed cases.

The daily new confirmed COVID-19 sequence data are modeled by smooth transition autoregressive model (STAR). The STAR model is divided into the autoregressive part and the nonlinear smooth part; the specific form of the model is as follows:

$$
\begin{aligned}
y_{t}= & \alpha_{0}+\alpha_{1} y_{t-1}+\cdots+\alpha_{p} y_{t-p} \\
& +\left(\beta_{0}+\beta_{1} y_{t-1}+\cdots+\beta_{p} y_{t-p}\right) G\left(\gamma, c ; y_{t-d}\right)+\varepsilon_{t},
\end{aligned}
$$

where $\left(\alpha_{0}, \alpha_{1}, \ldots, \alpha_{p}\right)$ and $\left(\beta_{0}, \beta_{1}, \ldots, \beta_{p}\right)$ are vector parameters, and $y_{t}$ is the response variable, $\left(y_{t-1}, \cdots, y_{t-p}\right)$ is the vector of the explanatory variables. $G\left(\gamma, c ; y_{t-d}\right)=1-\exp \left(-\gamma\left(y_{t-d}-c\right)^{2}\right)$, where $\gamma>0$ is the transition speed, and it is the function $G\left(\gamma, c ; y_{t-d}\right)$ that causes the nonlinearity of the model. It is continuous with the observable variable $y_{t-d}$. As $y_{t-d}$ changes, $G\left(\gamma, c ; y_{t-d}\right)$ changes between 0 and 1 smoothly. The parameter $\gamma>0$ determines the speed of the smooth transition. Moreover, parameter $c$ measures the position where the smooth transition takes place within the system under consideration, which can be viewed as the threshold for different growing patterns. Finally, $p$ is the order of time lag for autoregression, and $d$ is the time lag where state variable $y_{t-d}$ is converted.

In the application of the STAR model, all the parameters $p, d, \gamma, c, \alpha_{i}$, and $\beta_{i}, i=0,1, \ldots, p$, should be estimated, in which the values of $p$ and $d$ should be determined first, and then the remaining parameters are estimated. In this work, the time lag parameters are set as $p=7$ or $p=8$, and $d=4$ or $d=5$ for most cases due to the weekly stability of the time series and the statistical feature of incubation period.

The STAR model gives good fitting of the time series in consideration, and the associated nonlinear function $G$ tells the transition within the sequence. When a system is at a lower level, it is easy to cause an expansion, which makes the system expand; thus, $G\left(\gamma, c ; y_{t-d}\right)$ would decrease. On the other hand, when the system is at a higher level, it tends to decline; hence, the value of $G\left(\gamma, c ; y_{t-d}\right)$ would increase accordingly. The larger the value of $G\left(\gamma, c ; y_{t-d}\right)$ was, the more likely transition of expansion or contraction would take place. Therefore, the intrinsic change within the sequence of the daily confirmed cases can be obtained from the value of $G\left(\gamma, c ; y_{t-d}\right)$. The reference threshold for $G$ is usually 0.5 .

In order to evaluate the effect of the prevention measures, the comparison between the release time of a policy and the time that transition takes place in function $G$ can accomplish our task. When function $G$ goes across the threshold, intrinsic transition happens, and hence the 
effectiveness of policies is proven as long as the change in $G$ happens within a certain time span after the release of policies. Finally, the quantification of the error in our fitting result is the mean absolute value defined as

$$
\mathrm{MAE}=\frac{1}{n} \sum_{t=1}^{n}\left|y_{t}-\hat{y}_{t}\right|,
$$

where $\left\{y_{t}, t=1, \ldots, n\right\}$ is the time series of the real data and $\left\{\hat{y}_{t}, t=1, \ldots, n\right\}$ is the sequence of the fitted values from our model.

\section{Results}

According to the STAR model, the data of daily new cases in each region are fitted to the model. Moreover, the nonlinear function is obtained simultaneously to quantify the transition points within the sequence. The impact of policies on the epidemic will be analyzed by combining the transitions in the time series with the timeline of epidemic prevention policies released and implemented in different regions. Guangdong Province in China is analyzed first to show the validation of our STAR model and the positive impact of epidemic prevention policies in detail. Then, cases in different countries are analyzed afterwards. We focused on the effectiveness as well as the ineffectiveness conducted by typical countries. Moreover, we take a look at the second wave of COVID-19 in two European countries with our STAR model, resulting in calling for closer attention and more effective and stricter prevention policies.

\subsection{Effective Policies}

3.1.1. Guangdong Province of China. The Chinese government adopted a series of epidemic prevention policies decidedly, which have successfully contained the epidemic and set a successful experience. We selected the data of daily new confirmed cases from January 24 to March 2 in Guangdong Province, China. The moving average is set as 5 to smooth the fluctuation. The lag order for autoregression is set as $p=$ 3 due to the short epidemic period and the lag order for the nonlinear transition is set as $d=1$ due to the fast reaction. Then, the nonlinear fitting equation can be obtained as follows:

$$
\begin{aligned}
\hat{y}_{t}= & 76.6-4.2 y_{t-1}+7.6 y_{t-2}-3.8 y_{t-3} \\
& +\left(-76.8+5.7 y_{t-1}-8.0 y_{t-2}+3.6 y_{t-3}\right) \\
& \cdot\left[1-\exp \left(-24.0\left(y_{t-1}-50.8\right)^{2}\right)\right] .
\end{aligned}
$$

Figure 1 shows the real data after smoothness (blue solid line) and its fitted values by the STAR model (blue dashed line). The right axis shows the value of nonlinear functions $G$ changing with time (orange solid line) and the reference line with threshold 0.5 (orange dashed line). Given by the STAR model, the transitions within the sequence of daily new cases are obtained from function $G$.

To be specific, let us see the fitting results in more detail. The transition speed parameter $\gamma=24.0$ measures the speed of the structural transition, which indicates that the epidemic situation in Guangdong Province was characterized by rapid reaction. The duration of the transition within function $G$ is 2 weeks, which is the common time length for quarantine of susceptible people. $c=50.8$ indicates that about 50.8 new cases daily is the switch boundary of expansion and contraction. In fact, Guangdong had quickly adopted a series of antiepidemic measures during the period from January 24 to February 4 . The dates that policies released are represented in Figure 1 with vertical dashed lines. According to the STAR model, the changes in $G$ reflect the intrinsic changes within the time series.

The control policies are effective by comparing the changes in $G$ and the implementation of the policies shown in Figure 1. The effective policies in Guangdong Province represent cases in most Chinese provinces, showing that the epidemic can be quickly contained as long as epidemic prevention policies are timely and strictly conducted. The model fitting error is shown in Figure 2. The mean absolute error (MAE) for Guangdong is 2.3, which shows the good fitting of the STAR model. In the following, typical countries with effective containment policies are analyzed.

3.1.2. Italy. The daily new cases from February 22 to May 30, 2020 , were selected for Italy. The moving average order is set as 7 to smooth weekly fluctuation. The lag orders for autoregression and nonlinear function are set as $p=7$ and $d=5$, respectively. The nonlinear fitting equation is as follows:

$$
\begin{aligned}
\widehat{y}_{t}= & 92.4+1.2 y_{t-1}-0.1 y_{t-2}-0.2 y_{t-3} \\
& +0.4 y_{t-5}-0.4 y_{t-6}+0.1 y_{t-7} \\
& +\left(-62.9-0.2 y_{t-1}+0.3 y_{t-2}\right. \\
& \left.+0.1 y_{t-4}-0.1 y_{t-5}+0.2 y_{t-6}+0.5 y_{t-7}\right) \\
& \cdot\left[1-\exp \left(-5.3\left(y_{t-5}-3261.2\right)^{2}\right)\right] .
\end{aligned}
$$

The curves for the real data, fitting results, and $G$ function are shown in Figure 3. The smooth transition in $G$ takes place near $c=3261.2$, and the speed parameter is $\gamma=5.3$. The mean absolute error for Italy is 38.7 (Figure 4).

The impact of policies in Italy is positive. Compared to the fitting result of Guangdong Province with $\gamma=24.0$ and $c=50.8$, the speed for Italy is $\gamma=5.3$ and $c=3261.2$. Due to the large amount of daily confirmed cases, the boundary of transition is around 3261.2. It is difficult to prevent the pandemic when the scale is large. Therefore, the speed is slower. Since March 8, Italy has adopted extensive lockdown measures. Specific measures to restrict contacts were first implemented in the northern region and then conducted nationwide on March 10. As illustrated in Figure 3, the duration between two transitions indicated by function $G$ is nearly 1 month to get the daily new confirmed cases in Italy changing to shrinking period.

3.1.3. Germany. The data of daily new confirmed cases from February 28 to May 30, 2020, were selected for Germany. The moving average order is set as 7 to smooth the weekly periodic fluctuation. The lag orders for autoregression and nonlinear transition are set as $p=8$ 


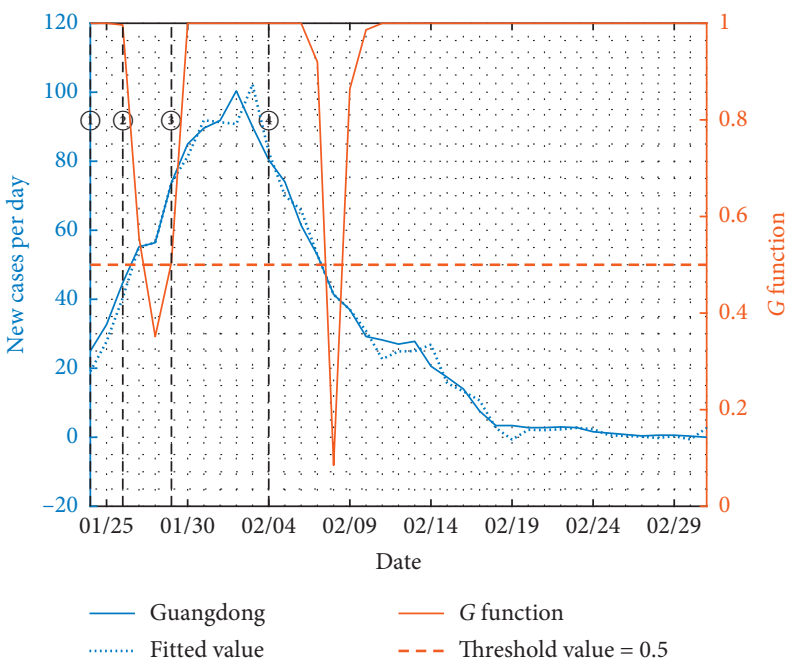

FIgURe 1: The fitting result of Guangdong Province, China. The left axis shows the daily confirmed cases for the real data after smoothness (blue solid line) and its fitted values by the STAR model (blue dashed line). The right axis shows the value of nonlinear functions $G$ changing with time (orange solid line) and the reference line with threshold 0.5 (orange dashed line). The vertical dotted line indicates the sequential released policies: (1) close entertainment and gatherings, (2) close other places, (3) isolate confirmed cases, and (4) suspend urban traffic.

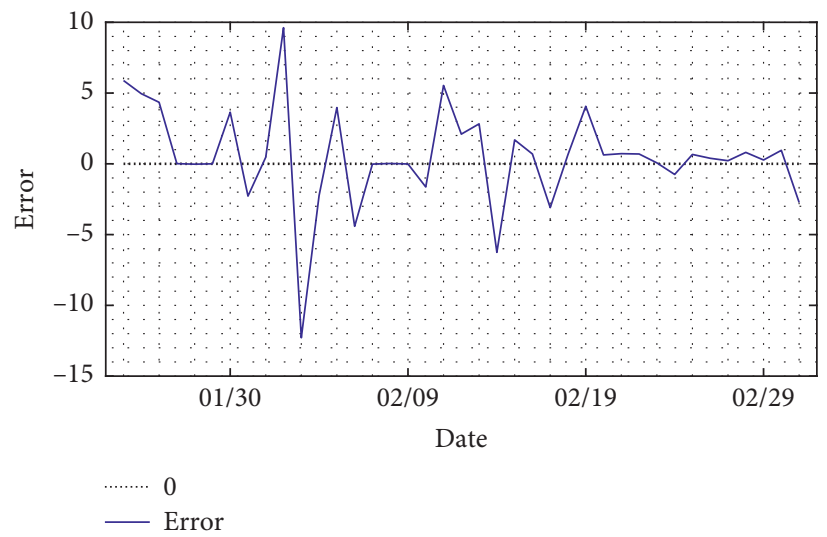

Figure 2: The fitting error of Guangdong Province, China. The mean absolute error is 2.3, indicating the goodness of fit.

and $d=4$, respectively. Then, we can get the nonlinear fitting equation as follows:

$$
\begin{aligned}
\widehat{y}_{t}= & -314.3+0.8 y_{t-1}+0.6 y_{t-2}-0.5 y_{t-3}+0.2 y_{t-4}-0.1 y_{t-5} \\
& +0.5 y_{t-6}-0.8 y_{t-7}+0.3 y_{t-8} \\
& +\left(358.0+0.5 y_{t-1}-0.9 y_{t-2}+0.7 y_{t-3}\right. \\
& \left.-0.1 y_{t-4}-0.3 y_{t-5}-0.5 y_{t-6}+0.4 y_{t-7}+1.7 y_{t-8}\right) \\
& \cdot\left[1-\exp \left(-2.2\left(y_{t-4}-2952.8\right)^{2}\right)\right] .
\end{aligned}
$$

The model fitting results and errors are shown in Figures 5 and 6 . The location of smooth transition is near $c=2952.8$, and the transition speed parameter is $\gamma=2.2$. The mean absolute error is 42 .

The transition speed parameter $\gamma=2.2$ shows that the containment in Germany is slow. Moreover, the transition boundary $c=2952.8$ indicates a severe situation. On March
8,2020 , the number of total confirmed cases in Germany exceeded 1,000. A week later, schools were closed. The government raised the risk level to "high." When the number of total confirmed cases exceeded 30,000 on March 23, the German government tightened social controls on activities and gatherings. The implementation of these policies made the daily confirmed cases decline and resulted in changes in $G$. The duration between the first and last transitions in $G$ is nearly 40 days, indicating the effectiveness of prevention and hard work for Germany to get the pandemic under control.

3.1.4. France. The data of daily new confirmed cases between February 26 and May 28, 2020, were selected for France, and the order of moving average is set as 7 to smooth the weekly periodic fluctuation. The lag orders for autoregression and nonlinear transition are set as $p=7$ and $d=4$, respectively. The nonlinear fitting equation is as follows: 


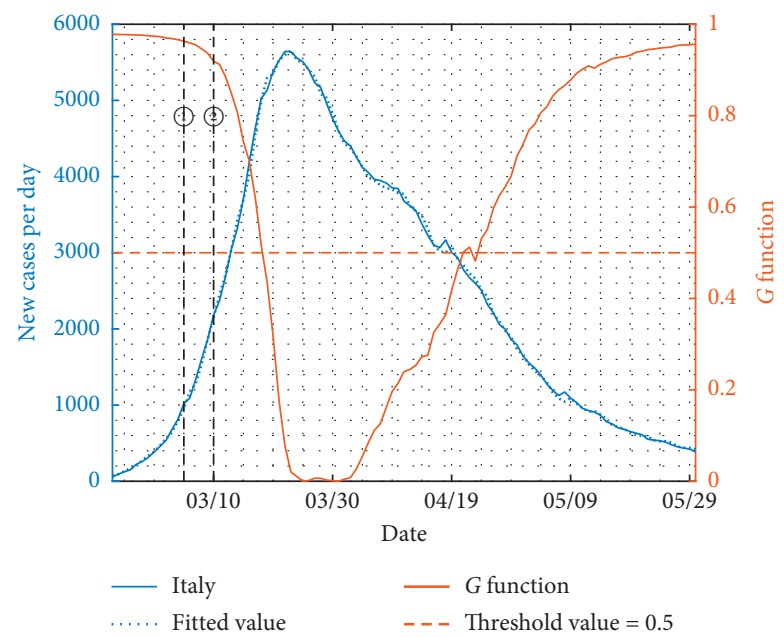

Figure 3: The fitting result of Italy. The left axis shows the daily confirmed cases for the real data after smoothness (blue solid line) and its fitted values by the STAR model (blue dashed line). The right axis shows the value of nonlinear functions $G$ changing with time (orange solid line) and the reference line with threshold 0.5 (orange dashed line). The vertical dotted line indicates the sequential released policies: (1) close schools across the country and (2) nationwide lockdown.

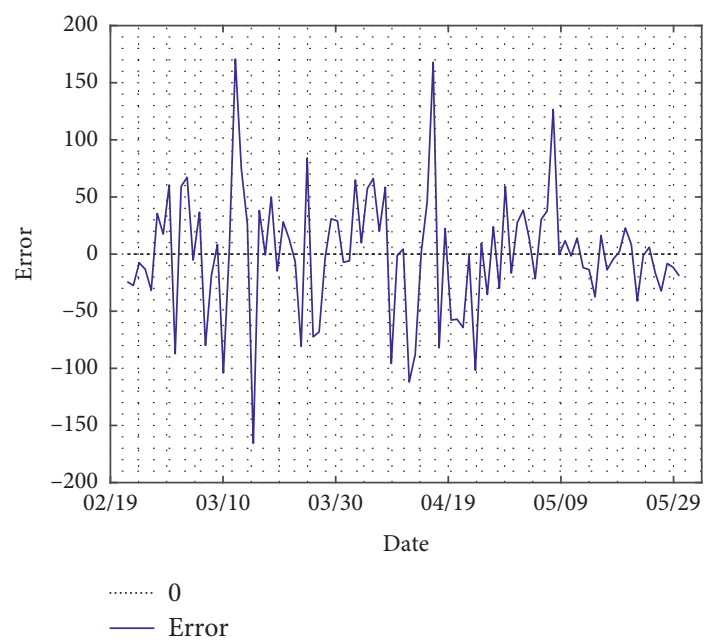

Figure 4: The fitting error of Italy with a mean absolute error of 38.7.

$$
\begin{aligned}
\widehat{y}_{t}= & 6728.3-1.7 y_{t-1}-0.7 y_{t-2}-0.4 y_{t-3}+1.0 y_{t-4} \\
& +1.4 y_{t-5}+4.5 y_{t-6}-3.4 y_{t-7}+\left(-6688.4+2.7 y_{t-1}+0.7 y_{t-2}+0.5 y_{t-3}-1.0 y_{t-4}-1.4 y_{t-5}-4.6 y_{t-6}+3.3 y_{t-7}\right) \\
& \cdot\left[1-\exp \left(-13.8\left(y_{t-4}-2890.3\right)^{2}\right)\right] .
\end{aligned}
$$

The fitting results and errors are shown in Figures 7 and 8. The location of smooth transition is near $c=2890.3$, and the transition speed parameter is $\gamma=13.8$. The mean absolute error is 96. The critical value for transition is $c=2890.3$, which is similar to that of Italy and Germany. The speed parameter $\gamma=13.8$, indicating a fast effectiveness of a series of policies released by the French government. In fact, the duration between the first and last transitions of $G$ is around 20 days, as shown in Figure 7, showing the fast and effective prevention measures in France.

3.1.5. Spain. The data of daily new confirmed cases for Spain were selected from February 25 to May 30, 2020. The moving average is set as 7 to smooth the weekly fluctuation. The lag orders for autoregression and nonlinear transition are set as 


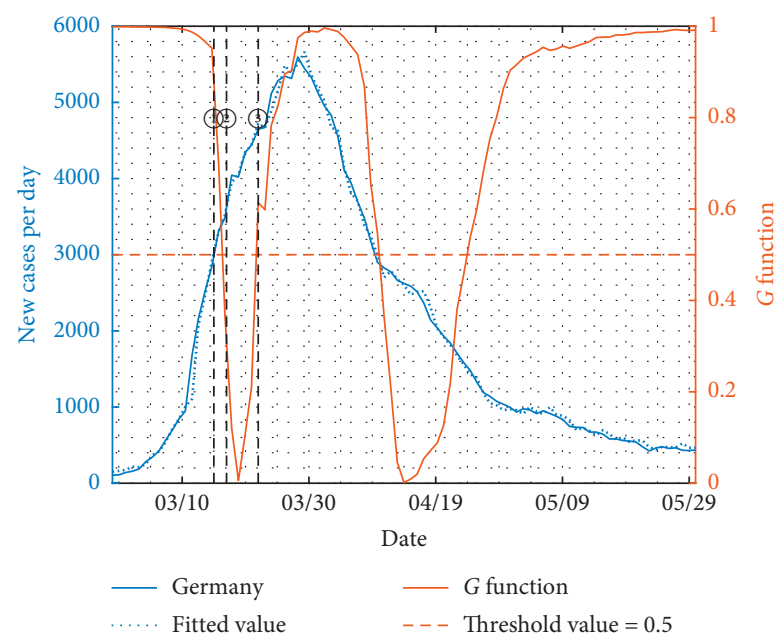

Figure 5: The fitting result of Germany. The left axis shows the daily confirmed cases for the real data after smoothness (blue solid line) and its fitted values by the STAR model (blue dashed line). The right axis shows the value of nonlinear functions $G$ changing with time (orange solid line) and the reference line with threshold 0.5 (orange dashed line). The vertical dotted line indicates the sequential released policies: (1) close schools across the country, (2) upgrade the risk level of the outbreak to "high," and (3) tighten controls on social activities and gatherings.

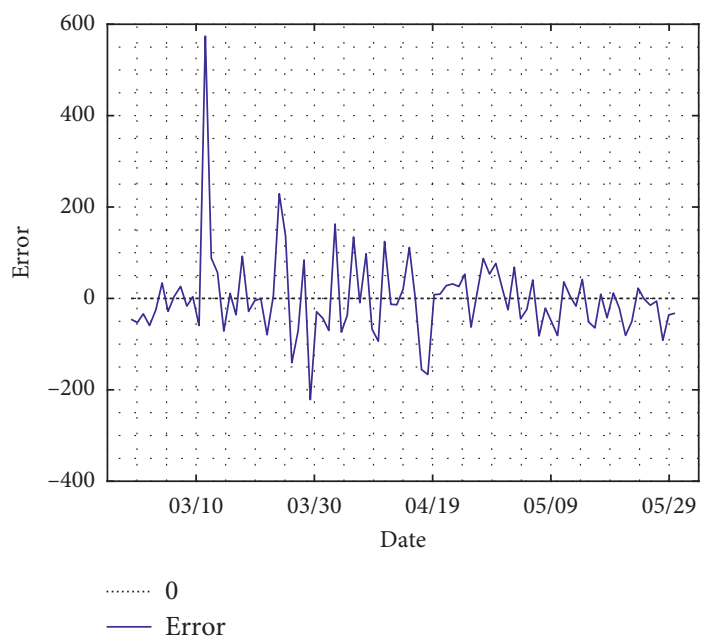

Figure 6: The fitting error of Germany with a mean absolute error of 42.

$p=7$ and $d=4$. Then, the nonlinear fitting equation is as

follows:

$$
\begin{aligned}
\widehat{y}_{t}= & 526.9+0.9 y_{t-1}-0.5 y_{t-2}+1.2 y_{t-3}-0.8 y_{t-4}+1.6 y_{t-5}-1.3 y_{t-6}-0.2 y_{t-7} \\
& +\left(-494.4+0.6 y_{t-1}+0.3 y_{t-2}-1.4 y_{t-3}+1.1 y_{t-4}-2.1 y_{t-5}+1.5 y_{t-6}+0.2 y_{t-7}\right)\left[1-\exp \left(-4.9\left(y_{t-4}-3618.1\right)^{2}\right)\right] .
\end{aligned}
$$

The model fitting results and errors are shown in Figures 9 and 10. The transition speed parameter is $\gamma=4.9$, indicating a slow transition and prevention effect. The boundary for smooth transition in Spain is around $c=3618.1$, showing a severe situation to combat the pandemic. The mean absolute error is 85 , showing a good fitting result.

Spain imposed a regional lockdown on March 16. Two weeks later, on March 30, Spain imposed a stricter restriction aiming at reducing mobility and nonessential industrial 


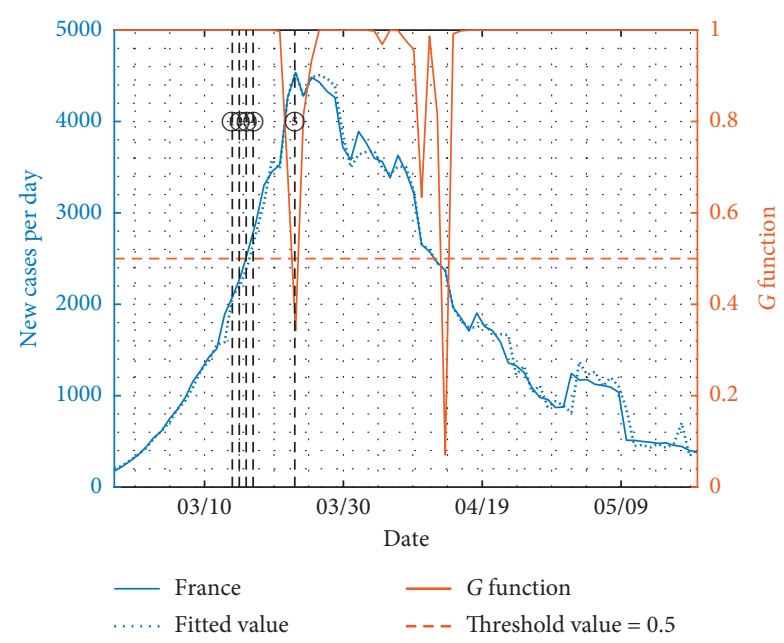

FIGURE 7: The fitting result of France. The left axis shows the daily confirmed cases for the real data after smoothness (blue solid line) and its fitted values by the STAR model (blue dashed line). The right axis shows the value of nonlinear functions $G$ changing with time (orange solid line) and the reference line with threshold 0.5 (orange dashed line). The vertical dotted line indicates the sequential released policies: (1) close all nonessential areas, (2) declare the highest stage of epidemic prevention and close all nonessential public places, (3) close all schools, (4) close borders, and (5) put field hospitals into operation.

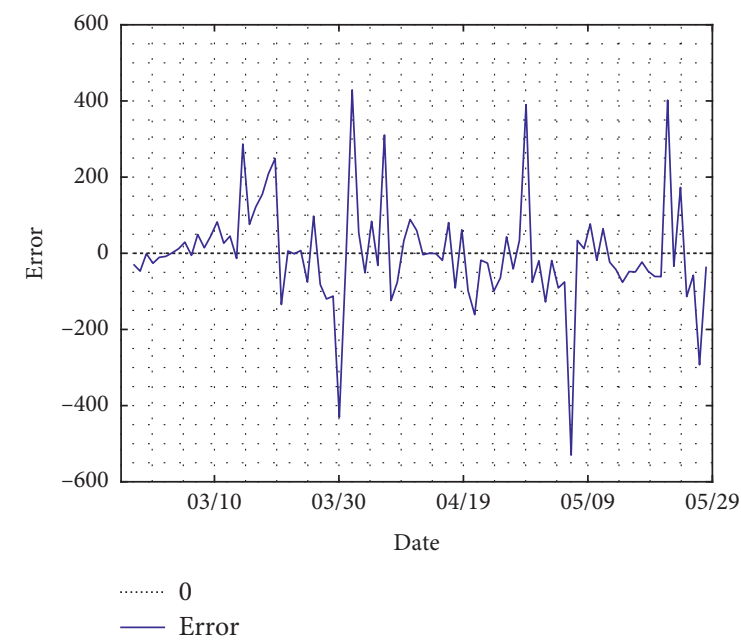

Figure 8: The fitting error of France with a mean absolute error of 96.

activity throughout the country. However, it took nearly two months for Spain to overcome the first wave of pandemic, resulting from the duration between the first and the last transitions of $G$. The transition speeds for Spain and Italy are 4.9 and 5.3, which are similar. However, it took nearly twice the time period for Spain to get the pandemic under control as that of Italy. The essence for the difference lies in the hesitation towards imposing strict restriction at early stage of the epidemic. The epidemic between March 16 and March

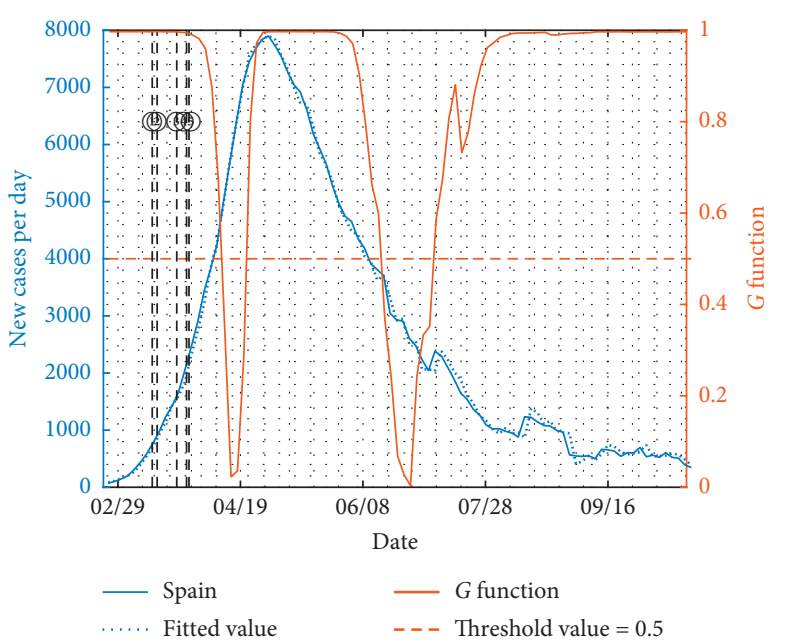

FIgURE 9: The fitting result of Spain. The left axis shows the daily confirmed cases for the real data after smoothness (blue solid line) and its fitted values by the STAR model (blue dashed line). The right axis shows the value of nonlinear functions $G$ changing with time (orange solid line) and the reference line with threshold 0.5 (orange dashed line). The vertical dotted line indicates the sequential released policies: (1) restrict free traveling for 15 days, (2) isolate residents at home and close nonessential places, (3) transform Madrid's convention and exhibition center into a "makeshift hospital," (4) force workers in noncore industries to stay at home, and (5) upgrade epidemic prevention and control measures.

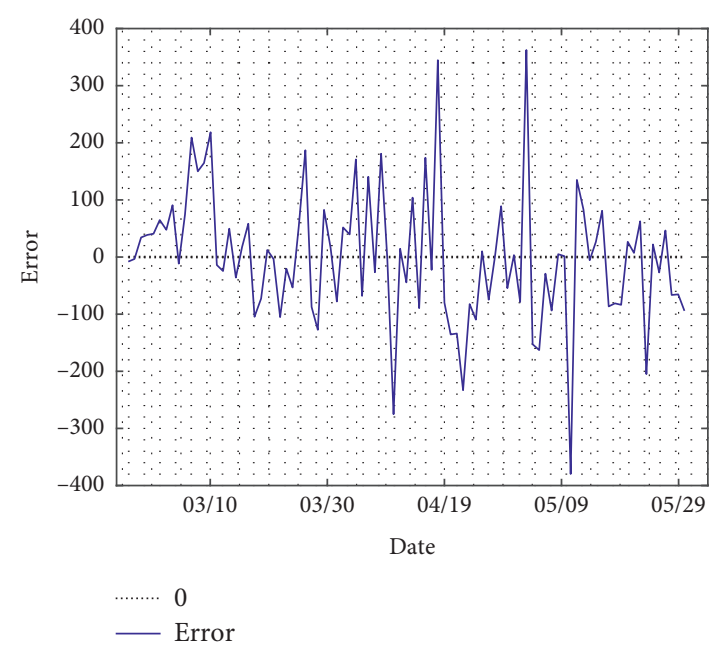

FIGURE 10: The fitting error of Spain with a mean absolute error of 85.

30 showed the ineffectiveness of the early control measures.

3.1.6. United States of America. The data of daily new confirmed cases for USA were selected from March 6 to May 24,2020 . The moving average order is set as 7 to smooth the weekly fluctuation. The lag orders for autoregression and nonlinear transition are set as $p=7$ and $d=4$, respectively. The nonlinear fitting equation is as follows: 


$$
\begin{aligned}
\hat{y}_{t}= & 34733.1-1.1 y_{t-1}-4.8 y_{t-2}+4.8 y_{t-3}-3.7 y_{t-4}-0.2 y_{t-5}-4.1 y_{t-6}+4.4 y_{t-7} \\
& +\left(-34058.6+2.0 y_{t-1}+4.8 y_{t-2}-4.9 y_{t-3}+4.8 y_{t-4}-0.3 y_{t-5}-4.3 y_{t-6}-4.0 y_{t-7}\right)\left[1-\exp \left(-4.5\left(y_{t-4}-15942.5\right)^{2}\right)\right] .
\end{aligned}
$$

The model fitting results and errors are shown in Figures 11 and 12. The transition speed parameter is $\gamma=4.8$, indicating a slow transition. The critical value for transition is around $c=15942.5$, reflecting a severe situation in USA. The mean absolute error for the fitting is 431 .

The nonlinear transition function $G$ in Figure 11 showed only one transition. The intrinsic contraction had not arrived till May 24, 2020. On April 17, the reopening policies were released aiming at recovering economy in three stages. There is no significant fluctuation short after the reopen. This indicates the possibility of reopening under close prevention. However, it is still at high risk of further outbreak since the system had not switched to contraction stage. As we know, after the death of Freud, the trend grew explosively due to demonstrations and gatherings. Close contacts made the transmission of coronavirus easy and resulted in further spread of the pandemic.

\subsection{Ineffective Policies}

3.2.1. Brazil. The data of daily new confirmed cases from March 5 to June 30, 2020, were selected for Brazil. The moving average order is set as 7 to smooth the weekly fluctuation. The lag orders of autoregression and nonlinear transition are set as $p=7$ and $d=4$, respectively. The nonlinear fitting equation is as follows:

$$
\begin{aligned}
\hat{y}_{t}= & 263.1+1.3 y_{t-1}-0.5 y_{t-2}-0.1 y_{t-3}+0.3 y_{t-5}+0.3 y_{t-6}-1.3 y_{t-7} \\
& +\left(-265.1-22.6 y_{t-1}+18.8 y_{t-2}+30.5 y_{t-3}+12.4 y_{t-4}-18.8 y_{t-5}-20.0 y_{t-6}+43.5 y_{t-7}\right)\left[1-\exp \left(-4.8\left(y_{t-4}-27722.5\right)^{2}\right)\right] .
\end{aligned}
$$

The model fitting results and errors are shown in Figures 13 and 14. The transition speed parameter is $\gamma=4.8$, indicating a slow transition. Due to the severe pandemic, the boundary between expansion and contraction is around $c=27722.5$. The mean absolute error is 272 .

In order to prove the effectiveness of certain policies, there must be changes within a period containing as short incubation period as possible. Since the common quarantine time is 14 days, the change should show up within 1 month or so. However, for the case of Brazil, not only the trend of the time series but also the $G$ function remained unaffected for more than 2 months after the release of policies. The STAR model shows a good fitting, as well as the ineffectiveness of the prevention measures.

3.2.2. India. The data of daily new confirmed cases were selected from March 3 to September 20, 2020, for India. The moving average is set as 7 to smooth the weekly periodic fluctuation. The lag orders of autoregression and nonlinear transition are set as $p=7$ and $d=4$, respectively. The nonlinear fitting equation is as follows:

$$
\begin{aligned}
\widehat{y}_{t}= & -23643.3+0.8 y_{t-1}+0.2 y_{t-2}-0.4 y_{t-3}-0.4 y_{t-4}-0.3 y_{t-5}+1.8 y_{t-6}-0.2 y_{t-7} \\
& +\left(23681.1+0.7 y_{t-1}-0.9 y_{t-2}+1.0 y_{t-3}-0.2 y_{t-4}+0.8 y_{t-5}-2.4 y_{t-6}+0.4 y_{t-7}\right)\left[1-\exp \left(-25.8\left(y_{t-4}-59008.5\right)^{2}\right)\right] .
\end{aligned}
$$

The model fitting results and errors are shown in Figures 15 and 16. The transition speed parameter is $\gamma=25.8$, indicating a quick transition. The critical value between expansion and contraction states is $c=59008.5$. The mean absolute error is 154 for our fitting.
The situation of India is similar to that of Brazil. However, it possesses longer time that the sequence trend and the transition function $G$ remained unaffected, which was more than 3 months, refer to Figure 15. The transition in $G$ indicated the slowdown of increase trend caused by potential natural growth. 


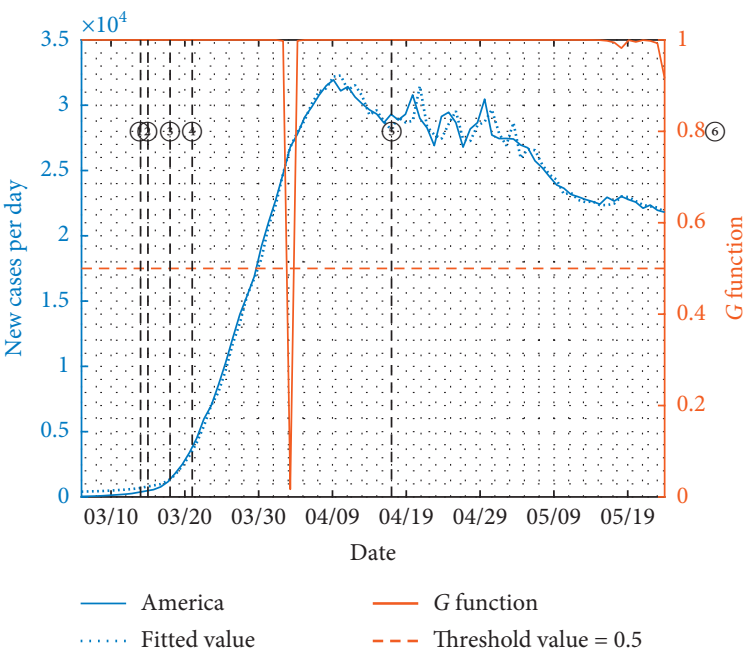

Figure 11: The fitting result of America. The left axis shows the daily confirmed cases for the real data after smoothness (blue solid line) and its fitted values by the STAR model (blue dashed line). The right axis shows the value of nonlinear functions $G$ changing with time (orange solid line) and the reference line with threshold 0.5 (orange dashed line). The vertical dotted line indicates the sequential released policies: (1) declare state of emergency and close schools, (2) impose travel ban to the United Kingdom and Ireland, (3) close the border with Canada, (4) close the border with Mexico, and (5) restart the US economy in three stages.

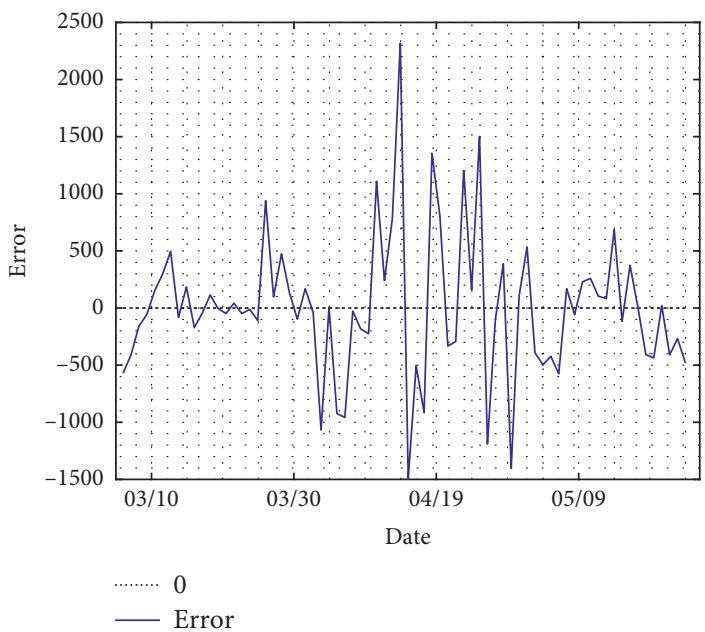

FIGURE 12: The fitting error of America with a mean absolute error of 431.

3.2.3. Mexico. The data of daily new confirmed cases from March 21 to July 10, 2020, were selected for Mexico. The moving average is set as 7 to smooth the weekly periodic fluctuation. The lag orders of autoregression and nonlinear transition are set as $p=7$ and $d=4$, respectively. The nonlinear fitting equation is as follows:

$$
\begin{aligned}
\hat{y}_{t}= & 217.8-0.1 y_{t-1}-0.4 y_{t-2}+1.1 y_{t-3}-1.1 y_{t-4}+1.4 y_{t-5}-1.0 y_{t-6}+1.3 y_{t-7} \\
& +\left(-192.8+1.6 y_{t-1}+0.1 y_{t-2}-1.4 y_{t-3}+1.5 y_{t-4}-1.9 y_{t-5}+1.4 y_{t-6}-1.5 y_{t-7}\right)\left[1-\exp \left(-32.0\left(y_{t-4}-3308.5\right)^{2}\right)\right] .
\end{aligned}
$$

The model fitting results and errors are shown in Figures 17 and 18. The transition speed parameter is $\gamma=32.0$, indicating a quick shift in the trend. The boundary for transition is $c=3308.5$. The mean absolute error is 22 . 


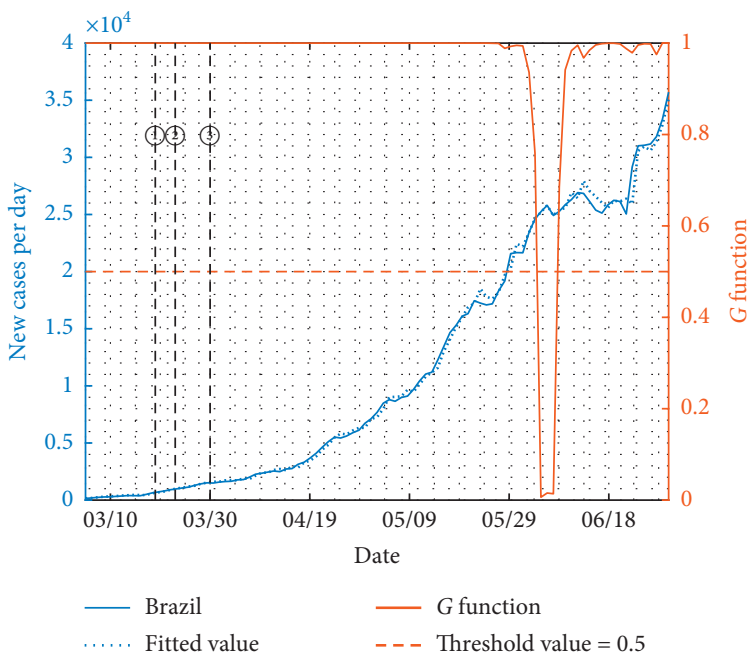

FIGURE 13: The fitting result of Brazil. The left axis shows the daily confirmed cases for the real data after smoothness (blue solid line) and its fitted values by the STAR model (blue dashed line). The right axis shows the value of nonlinear functions $G$ changing with time (orange solid line) and the reference line with threshold 0.5 (orange dashed line). The vertical dotted line indicates the sequential released policies: (1) close borders with Argentina, Bolivia, Colombia, Guyana, French Guiana, Paraguay, Peru, and Suriname within 15 days, (2) ban foreigners by air from China, European Union, Iceland, Norway, Switzerland, the United Kingdom, Australia, Japan, Malaysia, and South Korea, and (3) implement the lockdown policy.

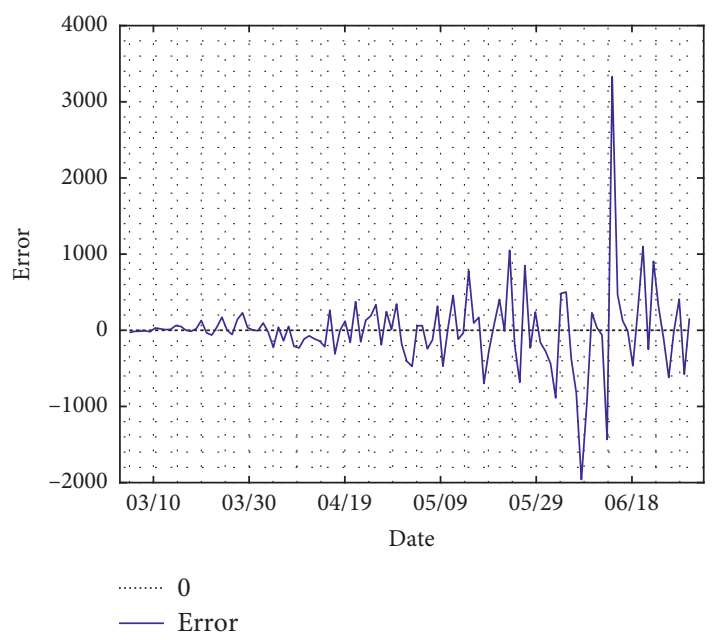

FIgURE 14: The fitting error of Brazil with an average absolute error of 272.

From Figure 17, there is no change for the trend sequence or function $G$ after 3 months since the release of the prevention policy, indicating the ineffectiveness of prevention measures in Mexico. The transition in $G$ in July indicated the slowdown of increase trend in daily new confirmed cases caused by potential natural growth.

3.3. Awareness of the Second Wave. The effective policies in most countries resulted in the temporary control of the COVID-19 pandemic and the reopen of economy and social activities. However, the second wave of the pandemic had been approaching dramatically. There was no awareness, and it was so fast that no measures had been conducted in time to contain the crazy growth. The pandemic had developed almost naturally without any intervention. In Figures 19 and 20, Italy and Germany are chosen to give an illustration of the gravity of the second wave by the STAR model.

As shown in Figures 19 and 20, the second wave has become severe since September. The $G$ function can only recognize the second wave in Italy due to the steep growth and large amount of daily new confirmed cases. For Germany, the STAR model shows the transitions in both waves 


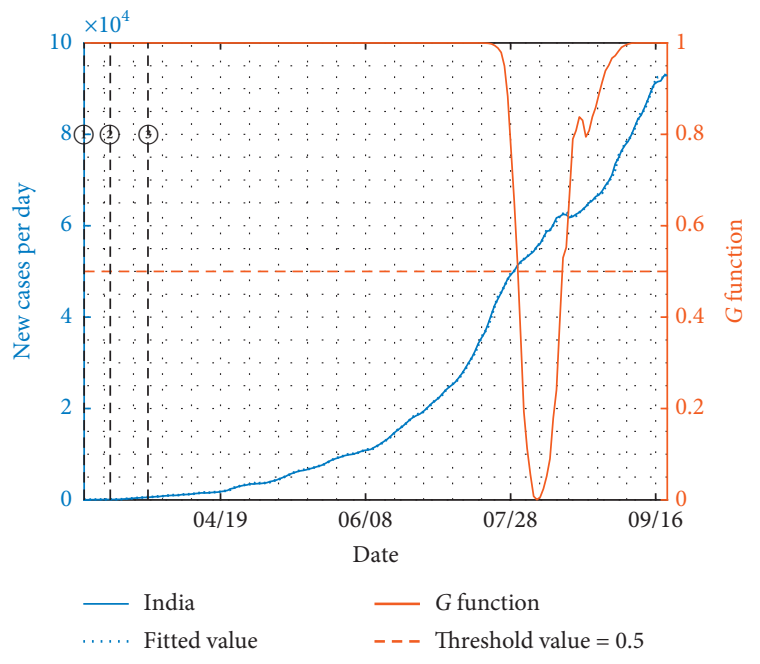

Figure 15: The fitting result of India. The left axis shows the daily confirmed cases for the real data after smoothness (blue solid line) and its fitted values by the STAR model (blue dashed line). The right axis shows the value of nonlinear functions $G$ changing with time (orange solid line) and the reference line with threshold 0.5 (orange dashed line). The vertical dotted line indicates the sequential released policies: (1) ban travellers from Italy, Iran, Korea, and Japan, (2) suspend visas of tourists, and (3) implement a lockdown policy.

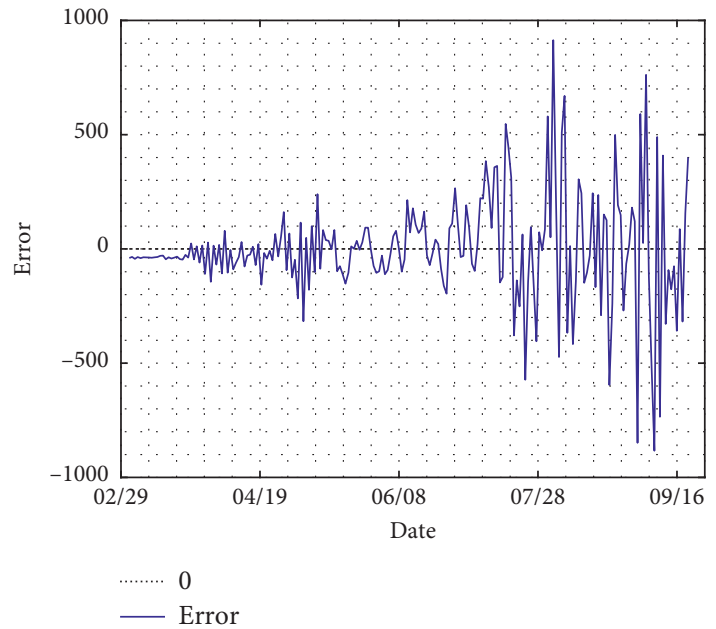

FIGURE 16: The fitting error of India with a mean absolute error of 154.

up to the ending date of our observation, which is October 27. In order to get the second wave under control, stricter measures and more effective policies are urgently needed.

\section{Discussion}

4.1. Effect of Prevention and Control Policies. We analyzed the daily new cases in 9 typical regions with the STAR model. Combining the release time of policies with the time series of daily new confirmed cases, it can be seen that effective policies would lead to a decrease in the number of daily

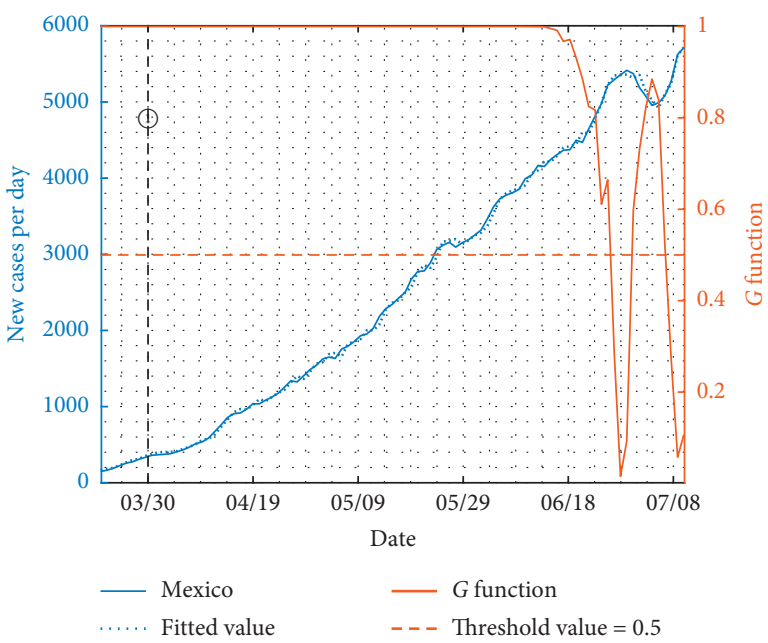

FIgURE 17: The fitting result of Mexico. The left axis shows the daily confirmed cases for the real data after smoothness (blue solid line) and its fitted values by the STAR model (blue dashed line). The right axis shows the value of nonlinear functions $G$ changing with time (orange solid line) and the reference line with threshold 0.5 (orange dashed line). The vertical dotted line indicates the sequential released policies: (1) declare the public health emergency and suspend nonessential activities until April 30.

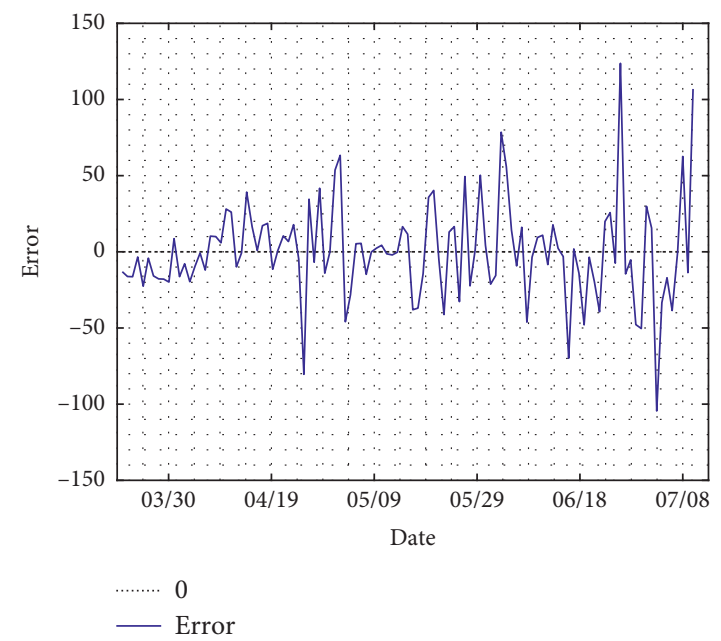

FIGURE 18: The fitting error of Mexico with a mean absolute error of 22.

confirmed cases within 2 weeks to 2 months since the release of certain policies. The inflection points and transitions between different stages of the epidemic curves illustrate the effectiveness of the policy. Meanwhile, in some countries, our model shows the ineffectiveness.

Policies usually include the restrictions on entry of travellers from countries with severe epidemic situations, the closure of national borders, and the suspension of travel. Moreover, schools and nonessential services are closed, large gatherings and public events are banned, and people were asked to stay at home. In the absence of vaccines, these prevention and control policies aiming at blocking transmission are necessary and effective. However, the 


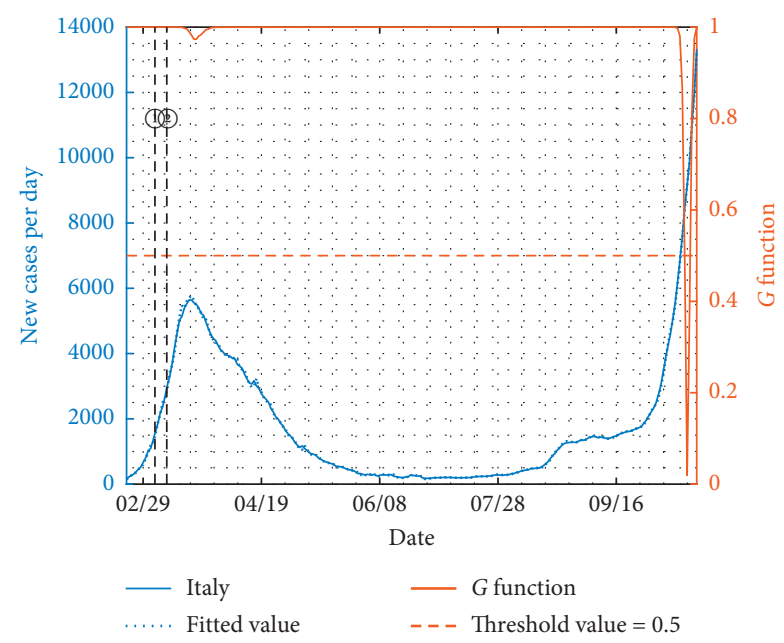

FIgURE 19: The fitting result of Italy containing the second wave. The left axis shows the daily confirmed cases for the real data after smoothness (blue solid line) and its fitted values by the STAR model (blue dashed line). The right axis shows the value of nonlinear functions $G$ changing with time (orange solid line) and the reference line with threshold 0.5 (orange dashed line). The vertical dotted line indicates the sequential released policies: (1) close schools across the country and (2) nationwide lockdown.

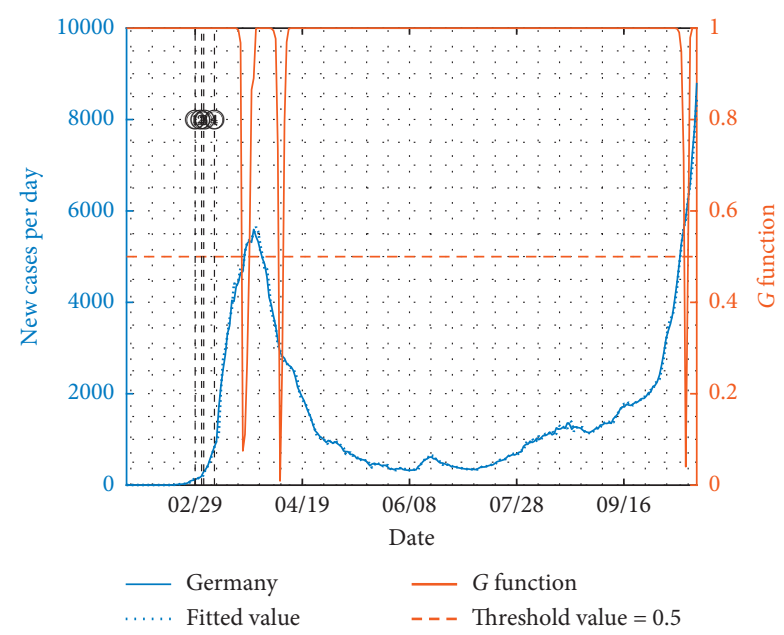

Figure 20: The fitting result of Germany containing the second wave. The left axis shows the daily confirmed cases for the real data after smoothness (blue solid line) and its fitted values by the STAR model (blue dashed line). The right axis shows the value of nonlinear functions $G$ changing with time (orange solid line) and the reference line with threshold 0.5 (orange dashed line). The vertical dotted line indicates the sequential released policies: (1) close schools across the country, (2) upgrade the risk level of the outbreak to "high," and (3) tighten controls on social activities and gatherings.

effectiveness depends on the implementation and cooperation of the masses.

4.2. Be Alert to the Second Wave. We can see from the epidemic curve that many countries are suffering from the second wave of the pandemic, which is more severe than the first wave. Faced with the social and economic pressure brought by the prevention of COVID-19, it is difficult to achieve a middle ground. Germany, France, UK, and Spain have already upgraded their prevention and containment measures for the current outbreak.

In short, governments should closely monitor the epidemic situation. Moreover, tracing close contacts, large-scale nucleic acid tests, and quarantining of travellers from regions of high risk are ways for preventing the potential pandemic in advance.

\section{Data Availability}

Data on daily confirmed COVID-19 cases were collected from the World Health Organization database (https://www.who.int/ data/collections; https://www.ecdc.europa.eu/en/publicationsdata/download-todays-data-geographic-distribution-covid-19cases-worldwide).

\section{Conflicts of Interest}

The authors declare that they have no conflicts of interest.

\section{Acknowledgments}

This work was jointly supported by the Fundamental Research Funds for the Central Universities (no. 210 2019XDA11) and the National Natural Science Foundation of China (Grant nos. 11971074, 61671005, 61372109, 61672108, and 61976025).

\section{References}

[1] C. Wang, P. W. Horby, F. G. Hayden, and G. F. Gao, "A novel coronavirus outbreak of global health concern," The Lancet, vol. 395, no. 10223, pp. 470-473, 2020.

[2] J. F.-W. Chan, S. Yuan, K.-H. Kok et al., "A familial cluster of pneumonia associated with the 2019 novel coronavirus indicating person-to-person transmission: a study of a family cluster," The Lancet, vol. 395, no. 10223, pp. 514-523, 2020.

[3] A. Lee, "Wuhan novel coronavirus (COVID-19): why global control is challenging?" Public Health, vol. 179, pp. A1-A2, 2020.

[4] Z. Du, X. Xu, Y. Wu, L. Wang, B. J Cowling, and L. A. Meyers, "Serial interval of COVID-19 among publicly reported confirmed cases," Emerging Infectious Diseases, vol. 26, 2020.

[5] Z. Yu, G. Zhang, Q. Liu, and Z. Lv, "The outbreak assessment and prediction of 2019-nCov based on thme-varying SIR model," Journal of University of Electronic Science and Technology of China, vol. 5, 2020.

[6] B. F. Maier and D. Brockmann, "Effective containment explains subexponential growth in recent confirmed COVID-19 cases in China," Science, vol. 368, no. 6492, p. 742, 2020.

[7] L. Hien, K. Veria, K. Piotr et al., "The positive impact of lockdown in Wuhan on containing the COVID-19 outbreak in China," Journal of Travel Medicine, vol. 27, 2020.

[8] J. T. Wu, K. Leung, and G. M. Leung, "Nowcasting and forecasting the potential domestic and international spread of the 2019-nCoV outbreak originating in Wuhan, China: a modelling study," The Lacent, vol. 395, 2020. 
[9] J. Hellewell, S. Abbott, G. Amy et al., "Feasibility of controlling COVID-19 outbreaks by isolation of cases and contacts," Lancet Glob Health, vol. 8, 2020.

[10] M. U. G. Kraemer, H. Tian, R. Li et al., "The effect of human mobility and control measures on the COVID-19 epidemic in China," Science, vol. 368, Article ID eabb4218, 2020.

[11] T. Zhou, Q. Liu, Z. Yang et al., "Preliminary prediction of the basic reproduction number of the Wuhan novel coronavirus 2019-nCoV," Journal of Evidence-Based Medicine, vol. 13, no. 1, pp. 3-7, 2020.

[12] S. Zhao, Q. Lin, J. Ran et al., "Preliminary estimation of the basic reproduction number of novel coronavirus (2019$\mathrm{nCoV}$ ) in China, from 2019 to 2020: a data-driven analysis in the early phase of the outbreak," International Journal of Infectious Diseases, vol. 92, 2020.

[13] Y. Zhang, "The epidemilogical characteristics of an outbreak of 2019 novel coronavirus distases (COVID-19) in China," Journal of Epidemiological, vol. 41, pp. 145-151, 2020.

[14] W. Guan, Z. Ni, L. Liu et al., "Clinical characteristics of coronavirus disease 2019 in China," The New England Journal of Medicine, vol. 382, 2020.

[15] G. A. Wellenius, S. Vispute, V. Espinosa et al., "Impacts of statelevel policies on social distancing in the united states using aggregated mobility data during the COVID-19 pandemic," 2020, http://arxiv.org/abs/2004.10172.

[16] M. Saez, A. Tobias, D. Varga, and M. A. Barceló, "Effectiveness of the measures to flatten the epidemic curve of COVID-19. The case of Spain," Science of The Total Environment, vol. 727, Article ID 138761, 2020.

[17] N. G. Davies, A. J. Kucharski, R. M. Eggo et al., "Effects of non-pharmaceutical interventions on COVID-19 cases, deaths, and demand for hospital services in the UK: a modelling study," Lancet Public Health, vol. 5, 2020.

[18] T. P. B. Thu, P. N. H. Ngoc, N. M. Hai, and L. A. Tuan, "Effect of the social distancing measures on the spread of COVID-19 in 10 highly infected countries," Science of The Total Environment, vol. 742, Article ID 140430, 2020.

[19] G. Chowell, C. Viboud, L. Simonsen, and S. M. Moghadas, "Characterizing the reproduction number of epidemics with early subexponential growth dynamics," Journal of The Royal Society Interface, vol. 13, no. 123, Article ID 20160659, 2016.

[20] L. Zhang, "Fitness of the generalized growth to the 2019 novel coronavirus data," Journal of University of Electronic Science and Technology of China, vol. 49, 2020.

[21] Q. Li, W. Feng, and Y.-H. Quan, "Trend and forecasting of the COVID-19 outbreak in China," Journal of Infection, vol. 80, no. 4, pp. 469-496, 2020.

[22] R. F. Engle, "Autoregressive conditional heteroscedasticity with estimates of the variance of United Kingdom inflation," Econometrica, vol. 50, no. 4, pp. 987-1007, 1982.

[23] J. D. Hamilton, "A new approach to the economic analysis of nonstationary time series and the business cycle," Econometrica, vol. 57, no. 2, pp. 357-384, 1989.

[24] C. Granger, T. Terasvirta, Modelling Nonlinear Economic Relationships, Oxford University Press, Oxford, UK, 1993.

[25] K. S. Chan, "On estimating thresholds in autoregressive models," Journal of Time, vol. 7, 1986. 